\title{
Nanoparticulated docetaxel exerts enhanced anticancer efficacy and overcomes existing limitations of traditional drugs
}

This article was published in the following Dove Press journal:

International Journal of Nanomedicine

29 September 2015

Number of times this article has been viewed

Jinhyang Choi, ${ }^{1,2}$ Eunjung Ko,' Hye-Kyung Chung, ' Jae Hee Lee,' Eun Jin Ju,' Hyun Kyung Lim, ${ }^{4}$ Intae Park, Kab-Sig Kim, ${ }^{5}$ Joo-Hwan Lee, ${ }^{5}$ Woo-Chan Son, ${ }^{6}$ Jung Shin Lee, ${ }^{1,7}$ Joohee Jung, ${ }^{1,4}$ Seong-Yun Jeong, ${ }^{1,2}$ Si Yeol Song, ${ }^{1,8}$ Eun Kyung Choi ${ }^{1,3,8}$

'Institute for Innovative Cancer Research, ${ }^{2}$ Asan Institute for Life Sciences, ${ }^{3}$ Center for Development and Commercialization of Anti-cancer Therapeutics, ${ }^{4}$ College of Pharmacy, Duksung Women's University, ${ }^{5}$ Bio-Synectics, ${ }^{6}$ Department of Pathology, ${ }^{7}$ Department of Internal Medicine, ${ }^{8}$ Department of Radiation Oncology, Asan Medical Center, University of Ulsan College of Medicine, Seoul, South Korea
Correspondence: Eun Kyung Choi Department of Radiation Oncology, Asan Medical Center, University of Ulsan College of Medicine, 88 Olympicro-43gil, Songpa-gu, Seoul 05505, South Korea

$\mathrm{Tel}+82230104432$

Fax +82 24867258

Email ekchoi@amc.seoul.kr

Seong-Yun Jeong

Asan Institute for Life Sciences, Asan Medical Center, University of Ulsan

College of Medicine, 88 Olympicro-43gil, Songpa-gu, Seoul 05505, South Korea

$\mathrm{Tel}+82230102648$

Fax+82230102619

Email syj@amc.seoul.kr
Abstract: Nanoparticulation of insoluble drugs improves dissolution rate, resulting in increased bioavailability that leads to increased stability, better efficacy, and reduced toxicity of drugs. Docetaxel (DTX), under the trade name Taxotere ${ }^{\mathrm{TM}}$, is one of the representative anticancer chemotherapeutic agents of this era. However, this highly lipophilic and insoluble drug has many adverse effects. Our novel and widely applicable nanoparticulation using fat and supercritical fluid (NUFS ${ }^{\mathrm{TM}}$ ) technology enabled successful nanoscale particulation of DTX (Nufs-DTX). Nufs-DTX showed enhanced dissolution rate and increased aqueous stability in water. After confirming the preserved mechanism of action of DTX, which targets microtubules, we showed that Nufs-DTX exhibited similar effects in proliferation and clonogenic assays using A549 cells. Interestingly, we observed that Nufs-DTX had a greater in vivo tumor growth delay effect on an A549 xenograft model than Taxotere ${ }^{\mathrm{TM}}$, which was in agreement with the improved drug accumulation in tumors according to the biodistribution result, and was caused by the enhanced permeability and retention (EPR) effect. Although both Nufs-DTX and Taxotere ${ }^{\mathrm{TM}}$ showed negative results for our administration dose in the hematologic toxicity test, Nufs-DTX showed much less toxicity than Taxotere ${ }^{\mathrm{TM}}$ in edema, paralysis, and paw-withdrawal latency on a hot plate analysis that are regarded as indicators of fluid retention, peripheral neuropathy, and thermal threshold, respectively, for toxicological tests. In summary, compared with Taxotere ${ }^{\mathrm{TM}}$, NufsDTX, which was generated by our new platform technology using lipid, supercritical fluid, and carbon dioxide $\left(\mathrm{CO}_{2}\right)$, maintained its biochemical properties as a cytotoxic agent and had better tumor targeting ability, better in vivo therapeutic effect, and less toxicity, thereby overcoming the current hurdles of traditional drugs.

Keywords: Nufs-DTX, docetaxel, anticancer efficacy, toxicity

\section{Introduction}

The application of nanotechnology to drug delivery has resulted in many significant advantages in pharmaceutical and biotechnology industries, such as improved delivery efficacy of slightly water-soluble drugs, greater stability and biocompatibility of drug products, extended route of administration, reduced toxicity, and effective targeted delivery to disease site. ${ }^{1,2}$ Engineered nanoparticles that are composed mainly of biological origin materials like lipids, phospholipids, chitosan, and dextran, or chemical materials like polymers, carbon, silica, and metals are the representative tools that have been proposed as effective carriers in this field. In addition, pure drugs themselves can be nanoparticulated to become carrier-free. ${ }^{1,3}$

Unlike engineered nanoparticles, which are generated as drug carriers and have a limitation, that is, secondary toxicity, nanoparticulation consisting of carrier-free, pure drug and minimal quantities of surface-stabilizing agents offers enhanced 
bioavailability, resulting in reduced toxicity and increased aqueous stability. ${ }^{3}$ Several nanoparticle engineering methods available in the pharmaceutical industry have been introduced and are being used commercially. ${ }^{4}$ Although conventional processes including wet milling and high pressure homogenization have been used for many years, several concerns still exist, such as contamination, batch variation, downstream processing difficulties, and compromised stability from long processing and high-energy milling. ${ }^{4}$ Alternatively, using supercritical fluids for nanoparticle formation have reduced the whole process to a single step. However, the supercritical fluid techniques have several limitations such as dependence on the efficiency of atomization of the solution. ${ }^{4}$ On the other hand, the spray-drying process, which is an alternative for nanoparticle formation, often needs organic solvent for the dissolution of drugs. ${ }^{5}$

Taxanes including paclitaxel (Taxol ${ }^{\mathrm{TM}}$ ) and docetaxel (DTX; Taxotere ${ }^{\mathrm{TM}}$ ) are remarkable cytotoxic compounds and are the representative chemotherapeutic anticancer drugs that are clinically well established. ${ }^{6}$ DTX, as well as paclitaxel, binds to microtubules and consequently stabilizes microtubules by enhancing the rates of nucleation, growth, and elongation phases of polymerization, leading to mitotic arrest, inhibition of cell proliferation, and stimulation of cell death. ${ }^{7}$ Taxanes are largely insoluble in water. DTX, which is a brown-yellow solution, is mixed in polysorbate 80 at a concentration of $40 \mathrm{~g} / \mathrm{L}$. This concentrate is first diluted in solvent $(13 \% \mathrm{w} / \mathrm{v}$ ethanol) followed by dilution in saline or dextrose saline. ${ }^{8}$ The high ratio of excipient in this commercialized DTX (DTX:excipient $=1: 33.8$ ) leads to various toxicities. The toxicity of taxanes is a well-known side effect and is broadly reported in taxane-treated patients. The most common toxic effects are hematopoietic suppression in bone marrow and neutropenia, which are regarded as common chemotherapy side effects. In particular, DTX leads to a high incidence of neurologic toxicities like peripheral nerve paralysis and fluid retention like peripheral edema. ${ }^{9}$

Nanoparticulation of soluble drugs with poor solubility using fat and supercritical fluid offers controlled size distribution and drug release, increased bioavailability, and reduction in toxicity. Our nanoparticulation method using fat and supercritical fluid (NUFS ${ }^{\mathrm{TM}}$ ) technology utilizes supercritical $\mathrm{CO}_{2}$ as the extracting solvent. Supercritical fluid extraction process is widely used in industrial scales either to remove unwanted materials from a product or to collect desired extracts. These simple and straightforward processes allow mass production of nano-sized particles up to a scale of several tons. Our NUFS ${ }^{\text {TM }}$ technology provides optimized delivery solution for poorly soluble drugs, such as smaller dosage, safer and more patientcompliant formulation, and avoidance of harsh excipients. Furthermore, unlike other pure drug-conjugated engineered nanoparticles with polymer, silica, and other metals, the problems of secondary toxicities caused by the materials of nanoparticles could be excluded with our technology. ${ }^{10-12}$ With enhanced dissolution rate and stability, it promises that more compounds can be evaluated for preclinical efficacy and safety. Additionally, this technology suggests increased cost-effectiveness and yield in early stage of development process.

To date, a number of studies introducing encapsulated taxanes, including Abraxane ${ }^{\circledR}$, which improve water solubility and delivery ability, have been reported. ${ }^{7,13-15}$ In this study, we formulated Nufs-DTX with a reduced ratio of DTX to excipient (DTX:excipient $=1: 0.95$ ) compared with Taxotere $^{\mathrm{TM}}$ and evaluated the antitumor effects of Nufs-DTX in vitro and in vivo. We observed dramatically increased dissolution rate and in vitro stability of Nufs-DTX in water. We also confirmed that the biochemical properties of NufsDTX on A549 cells as a cytotoxic agent were preserved. In addition, Nufs-DTX showed improved tumor accumulation in biodistribution studies and less toxicity than Taxotere ${ }^{\mathrm{TM}}$ in edema, paralysis, and paw-withdrawal latency that are regarded as fluid retention, peripheral neuropathy, and thermal threshold indicators, respectively, as toxicological tests.

\section{Materials and methods Preparation of DTX nanoparticle powder (Nufs-DTX powder)}

Docetaxel trihydrate (Shanghai Jinhe Bio-Technology Co., Ltd., Shanghai, People's Republic of China), polyvinylpyrrolidone (K17), and myristyl alcohol (BASF) were charged in a flask in the ratio of 1:0.8:10, and then melted by slowly heating to $100^{\circ} \mathrm{C}$. After the mixture was completely melted, it was cooled down at room temperature to obtain solid product.

The resulting solid product was charged in a pressureresistant reaction vessel. Then, while maintaining the temperature within the reaction vessel between $15^{\circ} \mathrm{C}$ and $20^{\circ} \mathrm{C}, \mathrm{CO}_{2}$ was added to elevate the pressure inside the reaction vessel to 60-90 atm. Under the temperature and pressure stated earlier, myristyl alcohol in the solid product was removed with flowing $\mathrm{CO}_{2}$ by controlling a vent valve of the vessel. Ultimately, the mixture powder 
of DTX and polyvinylpyrrolidone (K17) was obtained (Figure 1A).

The content of DTX in the mixture powder (ie, Nufs-DTX powder) was measured as $53.94 \% \pm 1 \%$. It was $1.62 \%$ lower than the theoretical value of $55.56 \%$. Based on this result, it was speculated that docetaxel trihydrate was changed to an anhydrous form during the melting and cooling process.

\section{Preparation of dispersion solution of DTX nanoparticles at $5 \mathrm{mg} / \mathrm{mL}$ (Nufs-DTX dispersion solution)}

In all, $36 \mathrm{mg}$ of Nufs-DTX powder was charged in a $10 \mathrm{~mL}$ vial and $3.7 \mathrm{~mL}$ of deionized water was added. The dispersion solution was then sonicated for 5 minutes at $40^{\circ} \mathrm{C}$ using bath type sonicator. Then, $200 \mu \mathrm{L}$ of poloxamer 188 solution (1\%, w/v; BASF SE, Ludwigshafen, Germany) and $100 \mu \mathrm{L}$ of polyoxyethylene 40 stearate solution $(1 \%, \mathrm{w} / \mathrm{v}$; Croda Europe Limited, East Yorkshire, UK) were added to the above dispersion solution. The resulting dispersion solution was then sonicated again for 15 minutes at $50^{\circ} \mathrm{C}$. The prepared Nufs-DTX dispersion solution was kept at room temperature until it was used in other experiments (Figure 1B).

\section{High-performance liquid chromatography analysis of DTX}

DTX concentration was measured at $230 \mathrm{~nm}$ by the highperformance liquid chromatography (HPLC) method (1525 Binary HPLC Pump and 2487 Dual Lambda Absorbance Detector; Waters Corporation, Milford, MA, USA). Samples were chromatographed on a $3.9 \times 250 \mathrm{~mm}$ reversed-phase stainless steel column packed with $5 \mu \mathrm{m}$ particles (XTerra RP18 Column, $5 \mu \mathrm{m}, 3.9 \times 150 \mathrm{~mm}$; Waters Corporation) and eluted with a mobile phase consisting of water/methanol/ acetonitrile $(42: 32: 26, \mathrm{v} / \mathrm{v})$ at a flow rate of $0.9 \mathrm{~mL} / \mathrm{min}$. The column temperature was maintained at room temperature. The samples were properly diluted with acetonitrile and directly injected $(20 \mu \mathrm{L})$ into the HPLC system without further treatment. The calibration curve of peak area against

A
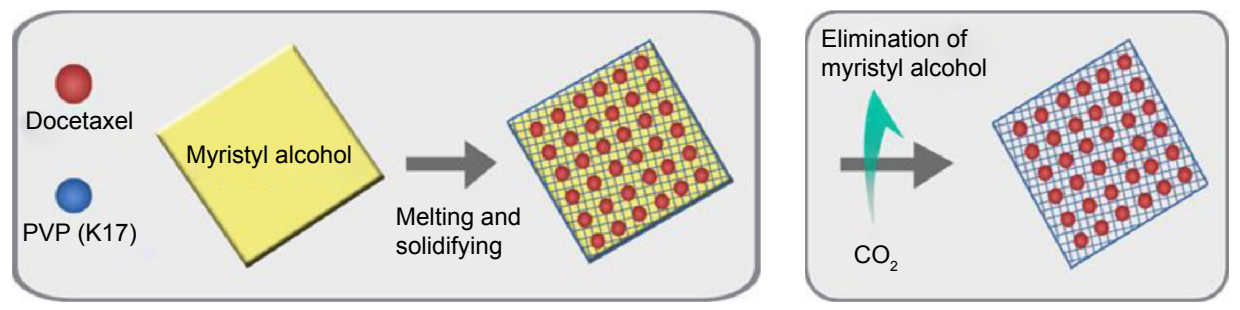

B

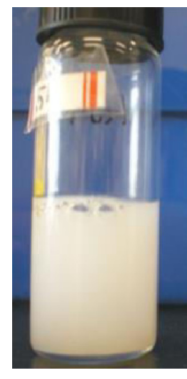

Size distribution by intensity

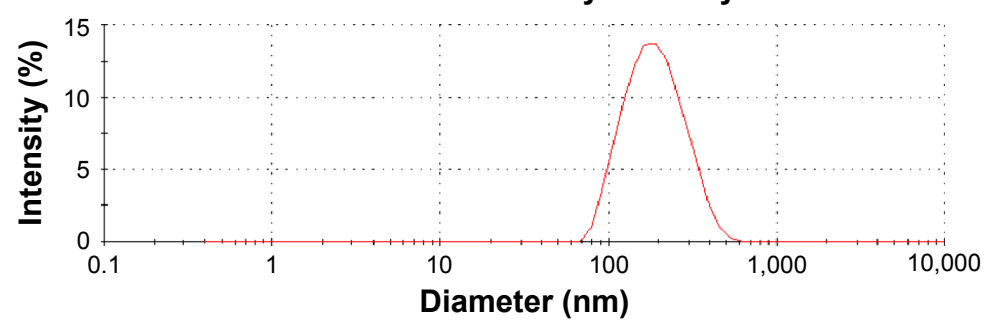

Nufs-DTX in $\mathrm{H}_{2} \mathrm{O}$

Nufs-DTX, 173.2 nm (PDI, 0.125)

C

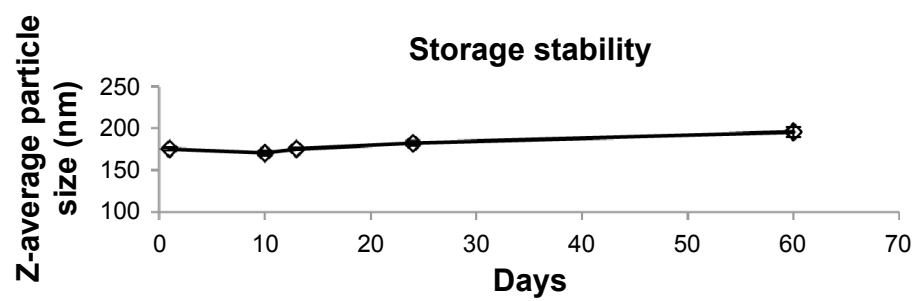

Figure I Physiochemical characterization of Nufs-DTX.

Notes: (A) A simple schematic of generation of Nufs-DTX. (B) The Nufs-DTX dispersion solution in DI water and size distribution by Zetasizer. (C) The physical stability of Nufs-DTX for 2 months at room temperature.

Abbreviations: DI, deionized; DTX, docetaxel; Nufs, nanoparticulation using fat and supercritical fluid; PVP, polyvinylpyrrolidone; PDI, polydispersity index. 
concentration of DTX was $\mathrm{A}=1,926.8 \mathrm{C}+3,272.5$ for the concentration range between 240 and $360 \mu \mathrm{g} / \mathrm{mL}\left(R^{2}=0.9991\right.$, where A indicates peak area and C indicates DTX indicates concentration).

\section{Characterization of Nufs-DTX dispersion solution}

The Z-average particle size and polydispersity index were determined by dynamic light scattering (Zetasizer Nano ZS, Malvern Instruments, Malvern, UK). All measurements were performed in at least triplicates at $25^{\circ} \mathrm{C}$. The $\mathrm{Z}$-average diameter of Taxotere ${ }^{\mathrm{TM}}$ in the Nufs-DTX dispersion was measured as $170 \pm 10 \mathrm{~nm}$ (Figure 1B).

\section{Cell culture and development of mouse models}

A549 (ATCC, Manassas, VA, USA) was cultured and maintained in F12K nutrient mixture (Kaighn's modification; Thermo Fisher Scientific, Waltham, MA, USA) containing $10 \%$ fetal bovine serum plus 100 units/mL penicillin and 100 $\mu \mathrm{g} / \mathrm{mL}$ streptomycin. Male Balb/c nude mice (5 weeks old) were purchased from SLC (Shizuoka, Japan) and were used to generate subcutaneous (sc) tumor mouse model as described in previous studies. ${ }^{16,17}$ All experiments were performed following the protocol approved by the Institutional Animal Care and Use Committee of ASAN Institute for Life Science. To generate an sc tumor mouse model, $1 \times 10^{6}$ A549 cells in $50 \mu \mathrm{L}$ of phosphate-buffered saline (PBS) were injected into sc tissues of the hind limb of each Balb/c nude mouse.

\section{Alpha-tubulin staining}

A549 cells were seeded on $10 \mathrm{~mm}$ round slide glasses and treated with Nufs-DTX or Taxotere ${ }^{\text {TM }}$ (as DTX; Sanofi S.A., Paris, France) at two different concentrations: 5 and $50 \mathrm{nM}$. After 2 and 16 hours of treatment, the slide glasses were washed with PBS ( $\mathrm{pH} 7.5$ ) and fixed in 4\% (w/v) paraformaldehyde. The fixed cells were permeabilized with 1:40 diluted Triton $\mathrm{X}-100$ solution and incubated with anti-alpha-tubulin (1:100; no. 2125; Cell Signaling Technology, Inc., Danvers, MA, USA) for 2 hours at $37^{\circ} \mathrm{C}$, and Alexa Fluor ${ }^{\circledR} 488$ donkey anti-rabbit secondary antibody (Jackson ImmunoResearch Laboratories, Inc., West Grove, PA, USA) for 1 hour at $37^{\circ} \mathrm{C}$. Vectashield mounting media with 4',6-diamidino-2-phenylindole (DAPI; Vector Laboratories, Burlingame, CA, USA) was used for mounting and counterstaining.

\section{Propidium iodide staining}

A549 cells were plated on a $100 \mathrm{~mm}$ tissue culture dish at a density of $5 \times 10^{5}$ and incubated overnight. After 24 hours of treatment with Nufs-DTX or Taxotere ${ }^{\text {TM }}$ at the IC50 concentration of $14.46 \mathrm{nM}$, the cells were collected and fixed by suspension in $70 \%(\mathrm{v} / \mathrm{v})$ ethanol. The fixed cells were stained with propidium iodide, and then cell cycle distribution was analyzed by flow cytometry. This experiment was performed in triplicates.

\section{Ki-67 staining}

A549 cells were cultured in a six-well plate coated with $0.1 \%$ gelatin. When cells were $80 \%$ confluent, they were treated with different concentrations of Taxotere ${ }^{\mathrm{TM}}$ or Nufs-DTX and incubated for 5 hours. Then, the cells were washed with PBS and incubated for 24 hours with new fresh media. After fixation with 4\% paraformaldehyde, Ki-67 antibody (Abcam, Cambridge, MA, USA) was applied. Alexa Fluor ${ }^{\circledR}$ 488 donkey anti-rabbit antibody (Jackson ImmunoResearch Laboratories, Inc.) was used as a secondary antibody. The fraction of proliferating cells was based on a count of cells in five random images taken from each case. The Ki-67 values were expressed as the percentage of positive cells in each case. This experiment was performed in triplicates.

\section{Clonogenic assay}

A549 cells were seeded into six-well plate at concentrations of 200-500 cells per well depending on the dose of Taxotere ${ }^{\mathrm{TM}}$ or Nufs-DTX. A549 cells were treated with Taxotere ${ }^{\text {TM }}$ or Nufs-DTX at the concentrations of $0.01,0.05$, or $0.1 \mathrm{ng} / \mathrm{mL}$. The cells were incubated for 10 days to allow for colony formation. The colonies composed of more than 50 cells were counted. Plating efficiency was defined from control cells as (mean colonies counted)/(cells plated). Survival fraction was calculated as (mean colonies counted) $/($ [cells plated $] \times$ [plating efficiency]). The assay was duplicated.

\section{In vivo distribution}

The A549 xenograft mouse model was grouped according to time of tumor mass harvest. Nufs-DTX or Taxotere ${ }^{\text {TM }}$ was administered intravenously (iv) through the tail vein at $60 \mathrm{mg} / \mathrm{kg}$ for each mouse. Each group had three mice $(\mathrm{n}=3$ per group). Whole blood, heart, lungs, liver, spleen, kidneys, and tumors were collected at each time point and kept in ice after weighing. All tissues were homogenized with $1 \mathrm{~mL}$ PBS and frozen until analysis. The Korea Clinical Research Center Co., Ltd. (Anyang, Korea) was requested to perform pharmacokinetic analyses for all the samples.

\section{Tumor growth delay}

Taxotere $^{\mathrm{TM}}$ or Nufs-DTX was administered iv through the tail vein into the A549 xenograft mouse at a concentration of 
$10 \mathrm{mg} / \mathrm{kg}$. A control group was treated with a concentration of $10 \%$ of dextrose solution, and an excipient group was treated with a concentration of $10 \mathrm{mg} / \mathrm{kg}$ of PVP K17. When the tumor volume reached $50 \mathrm{~mm}^{3}$, each agent was applied three times every 2 days. Sodium chloride solution ( $0.9 \%)$ was injected into a control group of animals. Numerical values of tumor size were recorded prior to each treatment. Each group had five mice ( $n=5$ per group). Tumor volume was measured regularly and calculated using the following formula:

$$
\mathrm{V}=(\text { longest dimension }) \times(\text { short dimension })^{2} \times 0.5
$$

\section{In vivo toxicity test}

The numbers of white blood cells (WBCs), platelets, or neutrophils were counted for the evaluation of hematologic toxicity, whereas aspartate transaminase (AST) and alanine transaminase (ALT) were measured for the evaluation of hepatotoxicity. To evaluate the function of the kidneys, blood urea nitrogen (BUN) and creatinine were measured. Rats ( 5 weeks old) were given a single injection of Taxotere ${ }^{\mathrm{TM}}$ or Nufs-DTX (10 mg/kg). At 1, 4, or 7 days after administration, samples of blood were taken from the abdominal vein. Blood was collected in Vacutainer blood collection tubes containing ethylenediaminetetraacetic acid (EDTA; Becton, Dickinson and Company, Franklin Lakes, NJ, USA) and stored in ice until hematologic analysis. This experiment was performed in triplicates.

For neurotoxicity and edema tests, male ICR mice (17-22 g, 4 weeks old) were purchased from Samtaco (Osan, Korea). The mice were acclimated in our animal facilities for 1 week and maintained in a temperature-controlled $\left(20^{\circ} \mathrm{C}-26^{\circ} \mathrm{C}\right)$ and light-controlled room (12-hour light-dark cycle). Mice were handled in accordance with the Guidelines of the Care and Use of Laboratory Animals, Duksung Women's University. Taxotere ${ }^{\mathrm{TM}}$ was used as a control drug. Nufs-DTX and Taxotere ${ }^{\mathrm{TM}}(5 \mathrm{mg} / \mathrm{mL})$ were diluted in sterile distilled water just before administration. Nufs-DTX and Taxotere $^{\mathrm{TM}}(10,15,20,25$, and $30 \mathrm{mg} / \mathrm{kg})$ were iv injected once a day for 5 consecutive days. The animals' conditions (behavior, breath, hair, skin, and feces) were recorded and body weights were measured daily during toxicity test.

Thermal nociceptive threshold was defined as the time required to lick the hind paw. This experiment was modified from previous studies. ${ }^{18,19}$ Briefly, the temperature was maintained at $48^{\circ} \mathrm{C}$, and the animals were placed on a hot plate (Ugo Basile, Varese, Italy). The cut-off time was 60 seconds. ${ }^{20}$

Paw edema was measured by an Ugo Basile plethysmometer (model 7140). The volume of paw was recorded just before the administration of Taxotere ${ }^{\mathrm{TM}}$ or Nufs-DTX as initial volume (Vo) in each mouse. Taxotere ${ }^{\mathrm{TM}}$, Nufs-DTX $(12.5,25,50$, and $75 \mathrm{mg} / \mathrm{kg}$ ), or saline (as control group) was iv injected at the same volume. After 30 minutes, the volume of paw (Vt) was measured. Edema (\%) was calculated by the following formula: ${ }^{21}$

$$
\operatorname{Edema}(\%)=\frac{\mathrm{Vt}(\mathrm{drug})-\mathrm{Vo}(\mathrm{drug})}{\mathrm{Vt}(\mathrm{control})-\mathrm{Vo}(\mathrm{control})} \times 100
$$

\section{Statistical analysis}

All data were expressed as mean \pm SD to represent at least three different experiments. Statistical analysis was performed by a Student's $t$-test or one-way analysis of variance using Prism statistical software (GraphPad Software, La Jolla, CA, USA). $P$-values less than 0.05 were considered statistically significant.

\section{Results}

\section{Stability of Nufs-DTX dispersion solution}

The preparation of Nufs-DTX powder is described in the Materials and Methods section (Figure 1A). The prepared Nufs-DTX powder was dispersed in deionized water by a protocol described in the Materials and Methods section, and the Nufs-DTX solution was kept at room temperature (Figure 1B). The physical stability of the Nufs-DTX dispersion solution was evaluated at $25^{\circ} \mathrm{C} \pm 2^{\circ} \mathrm{C}$. The changes in particle size were measured over a period of 2 months (Figure 1C). During this storage period, the particle size was not significantly changed, indicating that the NufsDTX dispersion solution is stable for at least 2 months. This indicates that Nufs-DTX with reduced excipient volume enhances water stability of insoluble drugs without any aggregation and size changes for a fairly long period in vitro. This promises convenience, ease of storage, and reduction in toxicities caused by harsh solvents such as polysorbate 80 and ethanol, which are used for hydrophobic Taxotere ${ }^{\mathrm{TM}}$ in clinics.

\section{Cellular response to Nufs-DTX}

We first evaluated the potential cytotoxic effects of NufsDTX in vitro. Taxotere ${ }^{\mathrm{TM}}$ was used as a positive control in all the experiments of this study. A549 cells were incubated with Nufs-DTX or Taxotere ${ }^{\mathrm{TM}}$ for 16 hours and stained with alpha-tubulin to verify excessive polymerization of microtubules. Similarly to Taxotere ${ }^{\mathrm{TM}}$, Nufs-DTX-treated A549 cells showed enhanced polymerization of the tubulin into microtubules and thereby induced formation of microtubule bundles (Figure 2A). Additionally, as taxanes are known 

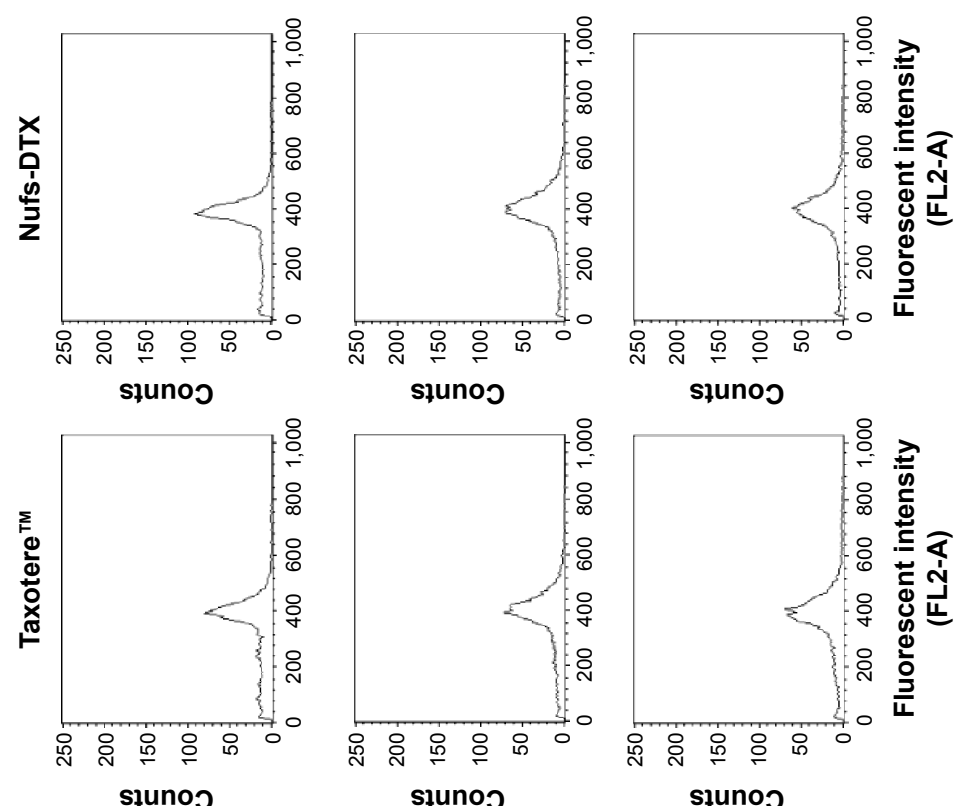

squnos
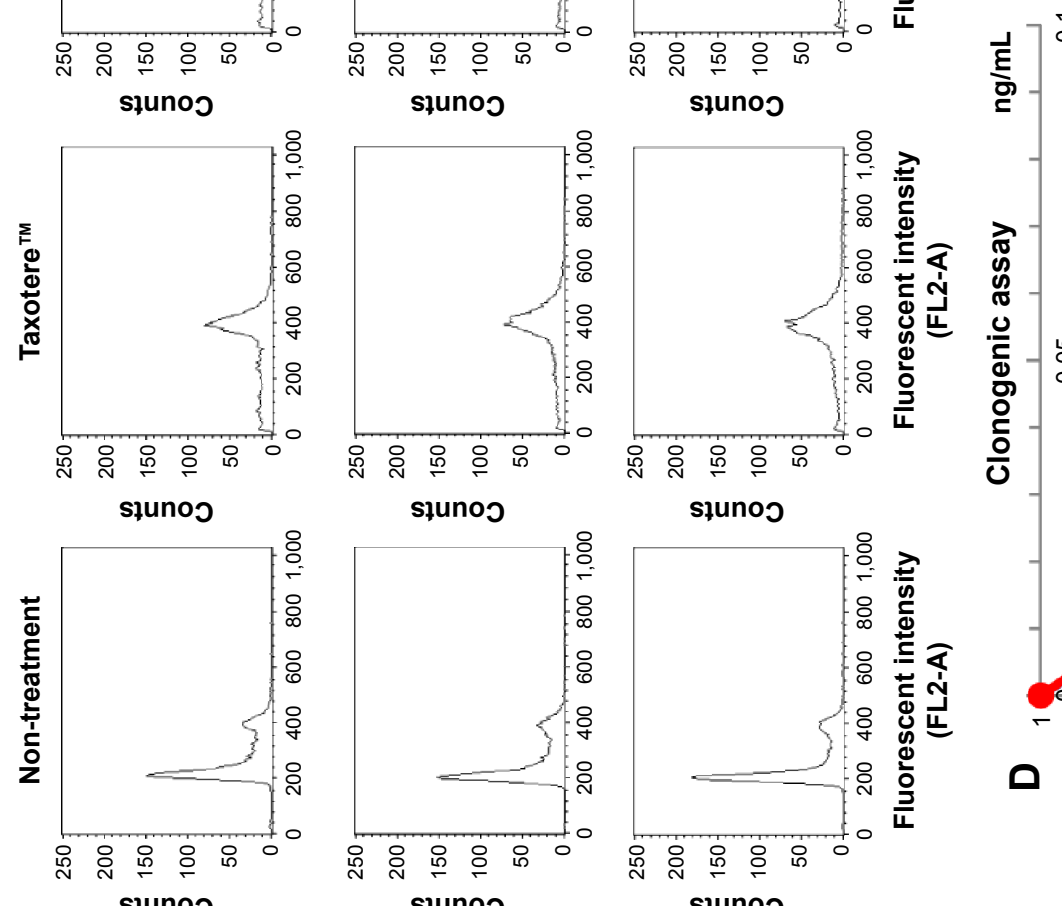

słunoo
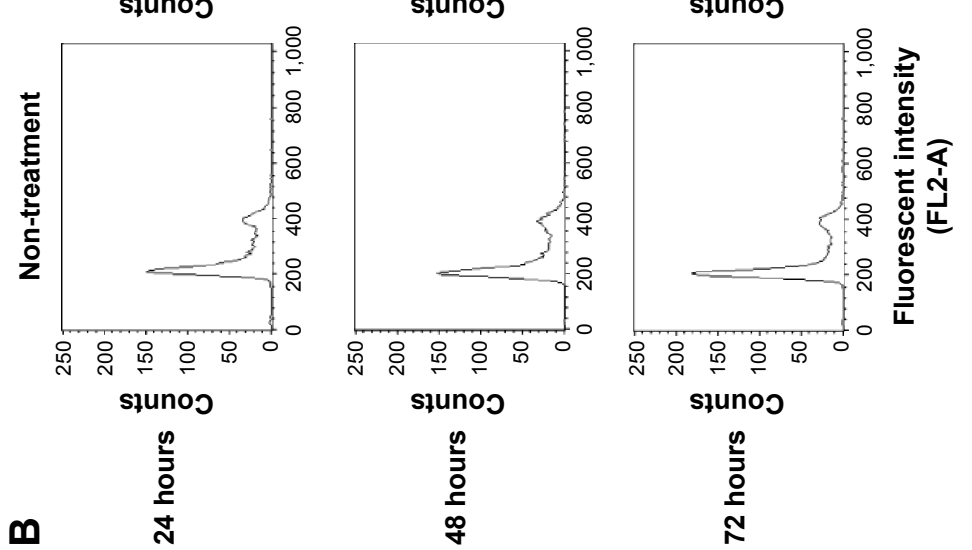

słunos

$$
\text { ำ }
$$
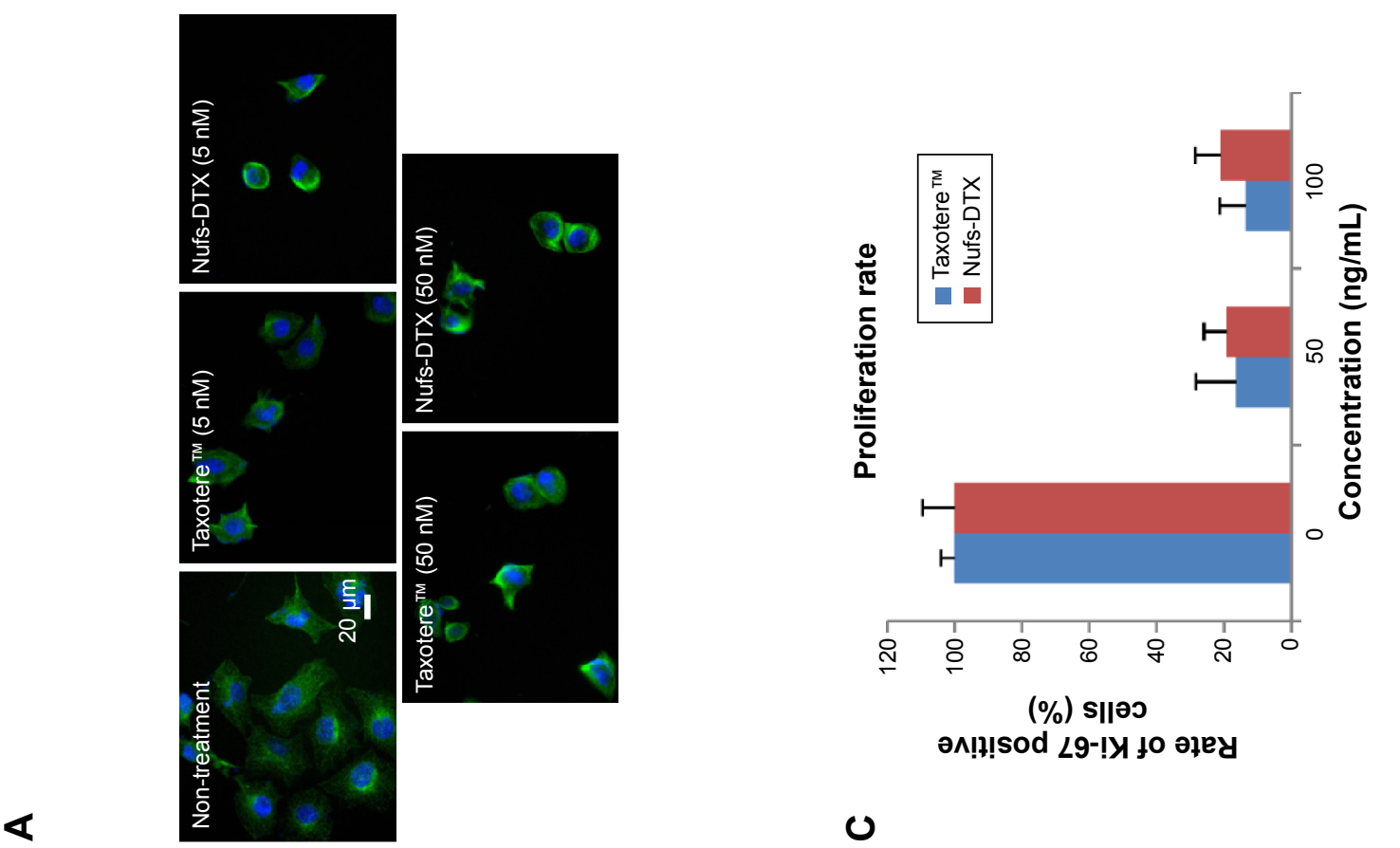

0

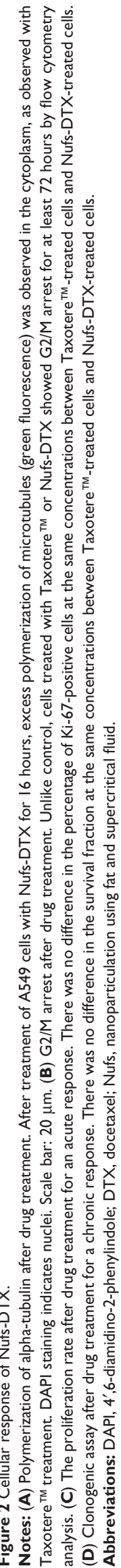


as mitotic inhibitors, we also observed that both the agents induced G2/M arrest in cells that were treated with the drugs (Figure 2B). This G2/M arrest was maintained for 72 hours. In order to confirm their cytotoxicity, we performed a Ki-67 assay for the short-term effect and a clonogenic assay for the long-term effect. The proliferation rate displayed by Ki-67 staining showed similar results in A549 cells treated with both the agents (Figure 2C). The proliferation rate of the Nufs-DTX-treated A549 cells was remarkably reduced at the concentration of $50 \mathrm{ng} / \mathrm{mL}$, and the reduction in proliferation rate was almost the same as that of the Taxotere ${ }^{\text {TM}}$-treated A549 cells. In addition, both the agents showed similar effects in clonogenic assay (Figure 2D). Taken together, we confirmed that the mechanism of the cytotoxic effect of Nufs-DTX was same as that of Taxotere ${ }^{\mathrm{TM}}$. In other words, Nufs-DTX preserves the pharmacologic action of DTX, which is known as a mitotic inhibitor that binds to tubulin and stabilizes microtubules.

\section{In vivo distribution and tumor growth delay of Nufs-DTX}

Biodistribution study is a routine strategy to check for improved drug efficacy and is performed at the earliest stages of new drug or drug delivery development. After tail vein injection of each agent at a concentration of $60 \mathrm{mg} / \mathrm{kg}$ into A549 sc xenograft mouse models, every important organ was collected and prepared for LC/MS analysis. Figure 3A shows the concentration of DTX residue in each organ at each collection time point. Based on our liquid chromatographymass spectrometry result, DTX was mainly detected in the lungs for at least 24 hours after administration, while small quantities were also detected in the liver, spleen, kidneys, and heart after Taxotere ${ }^{\mathrm{TM}}$ administration. Unfortunately, we did not observe any noticeable concentration of DTX in the tumors (Figure 3B) treated with Taxotere ${ }^{\mathrm{TM}}$. On the other hand, DTX was mainly detected in the liver for a short-term after administration and also in small quantities in the spleen, kidneys, and heart in the group treated with Nufs-DTX. Interestingly, we observed that DTX showed a faster clearance from the main organs in the group treated with Nufs-DTX (Figure 3A). Both the agents were rapidly cleared from the serum (Figure 3B).

In order to verify the in vivo therapeutic effect of Nufs-DTX, we explored its potential in tumor growth delay using an A549 sc xenograft mouse model. After treating the mice with each agent three times every other day, we observed that Nufs-DTX was the most effective drug for tumor growth delay compared with other groups (Figure 3C).
There were no changes in body weight in all the groups (data not shown).

Furthermore, we observed that DTX was accumulated in xenograft tumors at a relatively higher concentration after Nufs-DTX treatment compared with tumors from mice treated with Taxotere ${ }^{\mathrm{TM}}$ (Figure $3 \mathrm{~A}$ and $\mathrm{B}$ ). This phenomenon may be explained by the EPR effect, which is a property that tumor tissues obtain due to abundant newly generated abnormal blood vessels; $;^{22,23}$ nanoparticulated macromolecular drugs with large molecular weight have advantages in circulation time and accumulation in solid tumors. ${ }^{24}$ Anatomical abnormalities including extensive angiogenesis, high vascular density, defective vascular structure, slow venous return, and generation of permeability-enhancing factors, including vascular endothelial growth factors, bradykinin, peroxynitrite, prostaglandins, and matrix metalloproteinases, in solid tumor tissues lead to accentuated EPR effect resulting in increased accumulation of nanoparticulated drugs such as Nufs-DTX. ${ }^{24}$ Interestingly, we observed that there was no difference between Taxotere ${ }^{\mathrm{TM}}$ and Nufs-DTX in their serum concentrations of DTX at different time points (Figure 3B). In parallel to the distribution test results, Nufs-DTX reduced the speed of tumor growth more effectively than Taxotere ${ }^{\mathrm{TM}}$ and control (Figure 3C).

\section{In vivo toxicity of Nufs-DTX}

Engineered nanoparticles that are generated to improve the delivery of free drugs to target sites in human body have raised a number of concerns regarding the toxicity provoked by the nanoparticle materials. Alternatively, nanoparticulation of free drugs themselves reduces the toxicity caused by carrier materials. Therefore, we confirmed the feasibility of Nufs-DTX as an antitumor chemotherapeutic agent via in vivo toxicity assay. First of all, we evaluated the complete blood count for septicemia from blood samples of rats treated with each agent. We collected the blood samples at 1, 4, or 7 days posttreatment. When compared with a negative control, blood samples from mice that were treated with either Taxotere $^{\mathrm{TM}}$ or Nufs-DTX showed slightly lower numbers of WBCs, neutrophils, or platelets. However, these variations were either within the normal range or did not excessively exceed the normal range (Figure 4A). This result reflects our previous observations, which showed a fast clearance of both Nufs-DTX and Taxotere ${ }^{\mathrm{TM}}$. We additionally performed AST and ALT tests for hepatotoxicity and BUN and creatinine tests for nephrotoxicity. All the values from the groups treated with each agent were within the normal range for our experimental concentrations (Figure 4B). Interestingly, we did not 
A

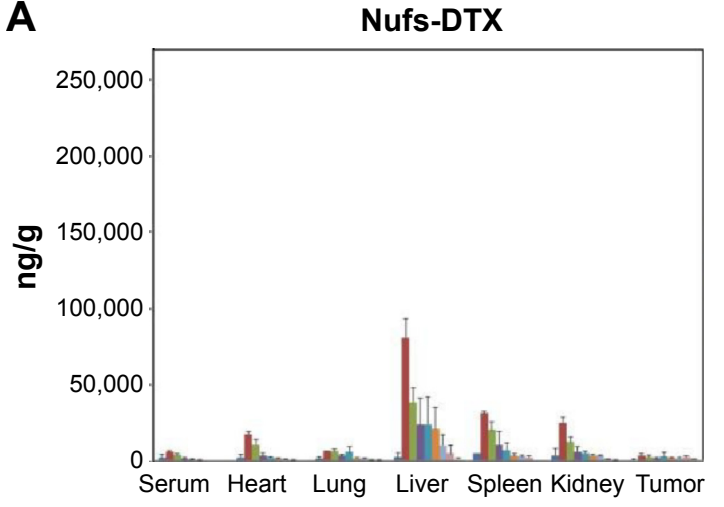

Taxotere $^{\mathrm{TM}}$

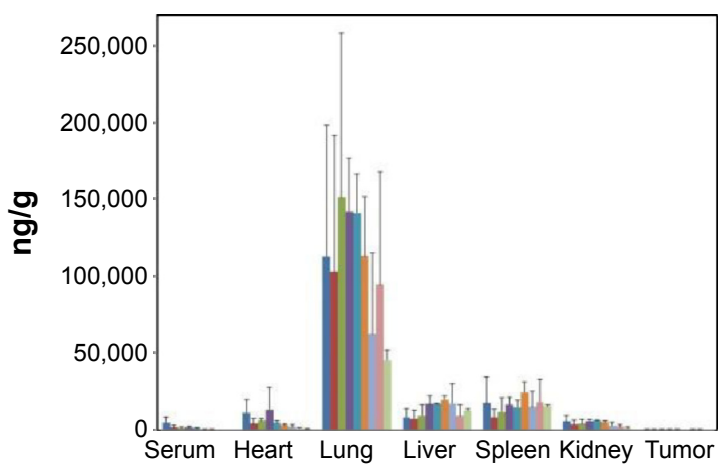

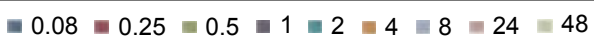

B
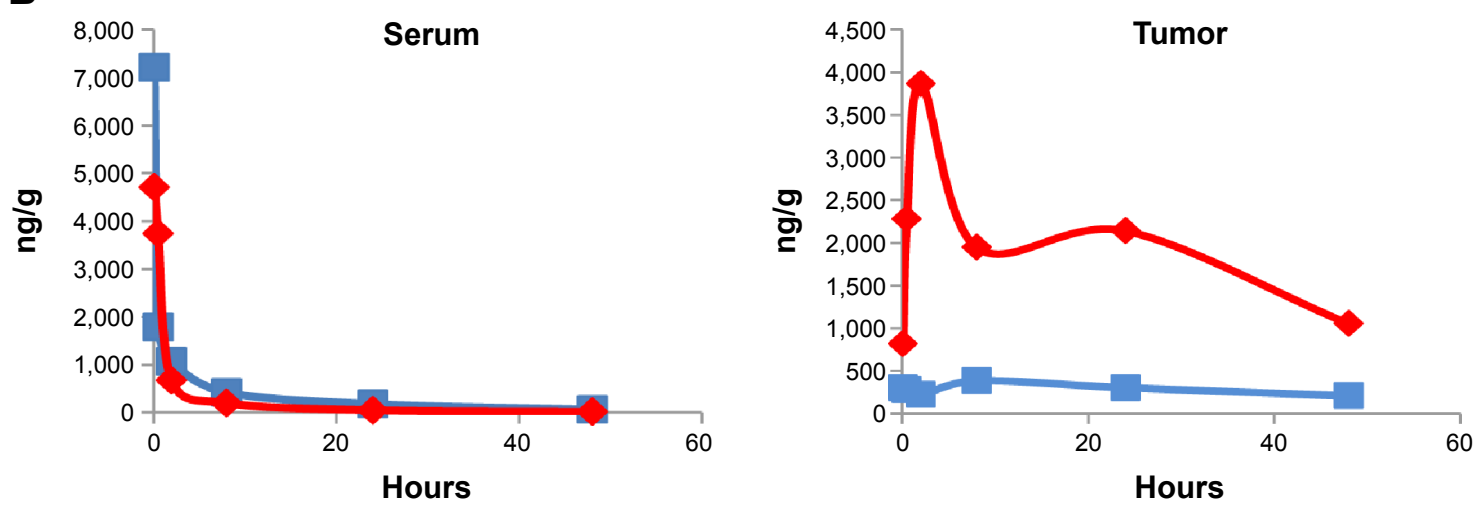

$=$ Taxotere $^{\mathrm{TM}} \longrightarrow$ Nufs-DTX

C

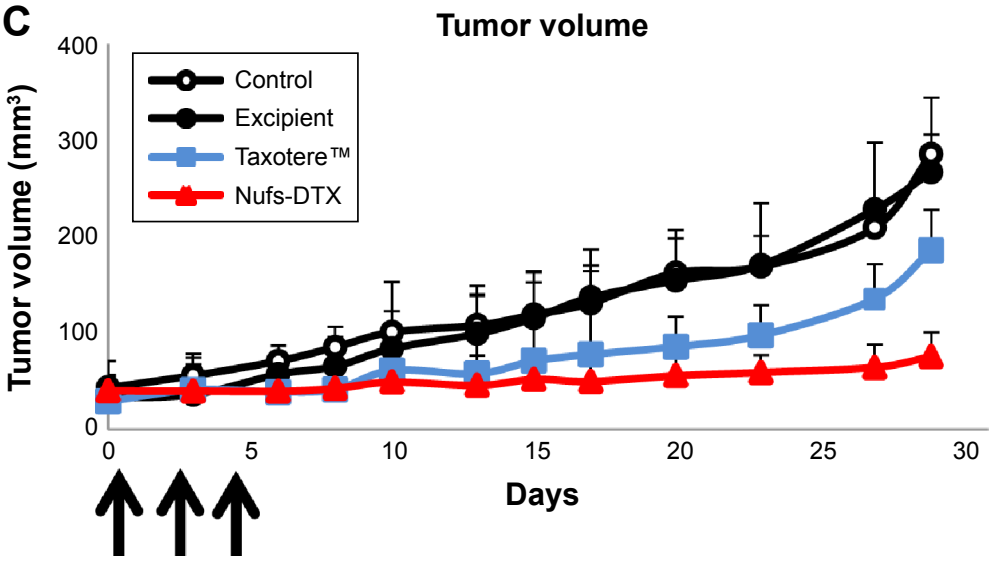

Figure 3 In vivo distribution and tumor growth delay of Nufs-DTX.

Notes: (A) Tissue distribution of Nufs-DTX after intravenous injection. Unlike Taxotere ${ }^{\mathrm{TM}}$ that was accumulated mainly in the lungs after intravenous administration, NufsDTX was observed mainly in the liver. Taxotere ${ }^{T M}$ was detected in the main organs including the lungs, liver, spleen, and kidneys over the collecting time points (hour), while Nufs-DTX was well cleared from each organ. (B) In serum, both the drugs showed similar pattern in distribution and clearance over a period of time. In tumors, a relatively large amount of accumulated Nufs-DTX was observed compared with Taxotere ${ }^{\mathrm{TM}}$. (C) Tumor growth delay after injecting three times every other day. Nufs-DTX showed effective tumor growth delay compared with other controls, including Taxotere ${ }^{\mathrm{TM}}$-treated group. Arrows indicate time of injections.

Abbreviations: DTX, docetaxel; Nufs, nanoparticulation using fat and supercritical fluid.

observe any hepatotoxicity in spite of the high accumulation of DTX in the liver, as shown by the distribution results of the Nufs-DTX-treated group (Figure 3A). This supposedly indicates that Nufs-DTX was not sufficiently solubilized in the liver to display any efficacy or toxicity.
DTX-induced peripheral neuropathy was frequently reported. ${ }^{25,26}$ Hence, we used our Nufs-DTX to investigate whether DTX-induced neuropathy is attenuated. We used high doses in order to compare the toxicities of Nufs-DTX with Taxotere ${ }^{\mathrm{TM}}$. As a result, the mice in the Taxotere ${ }^{\mathrm{TM}}$-treated 

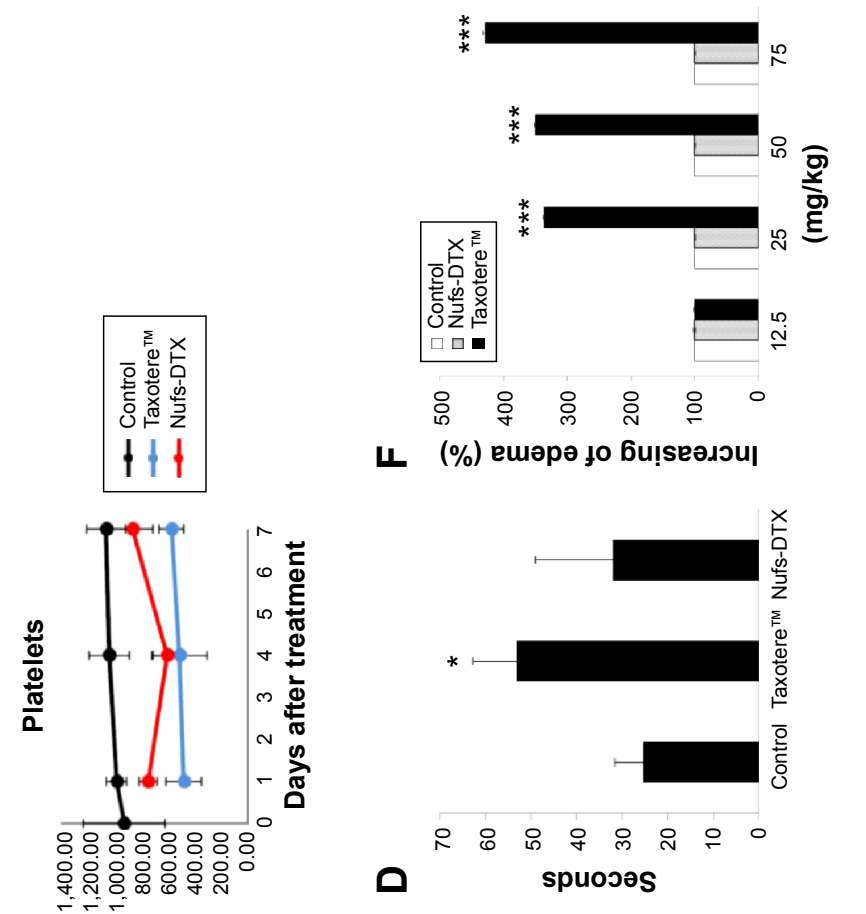

ᄂ. (\%) ешәрә до би!seәдэи

ךr/s||əo عOLX

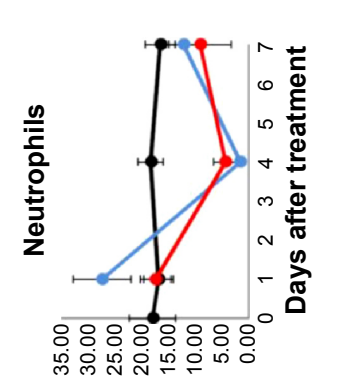

$\%$
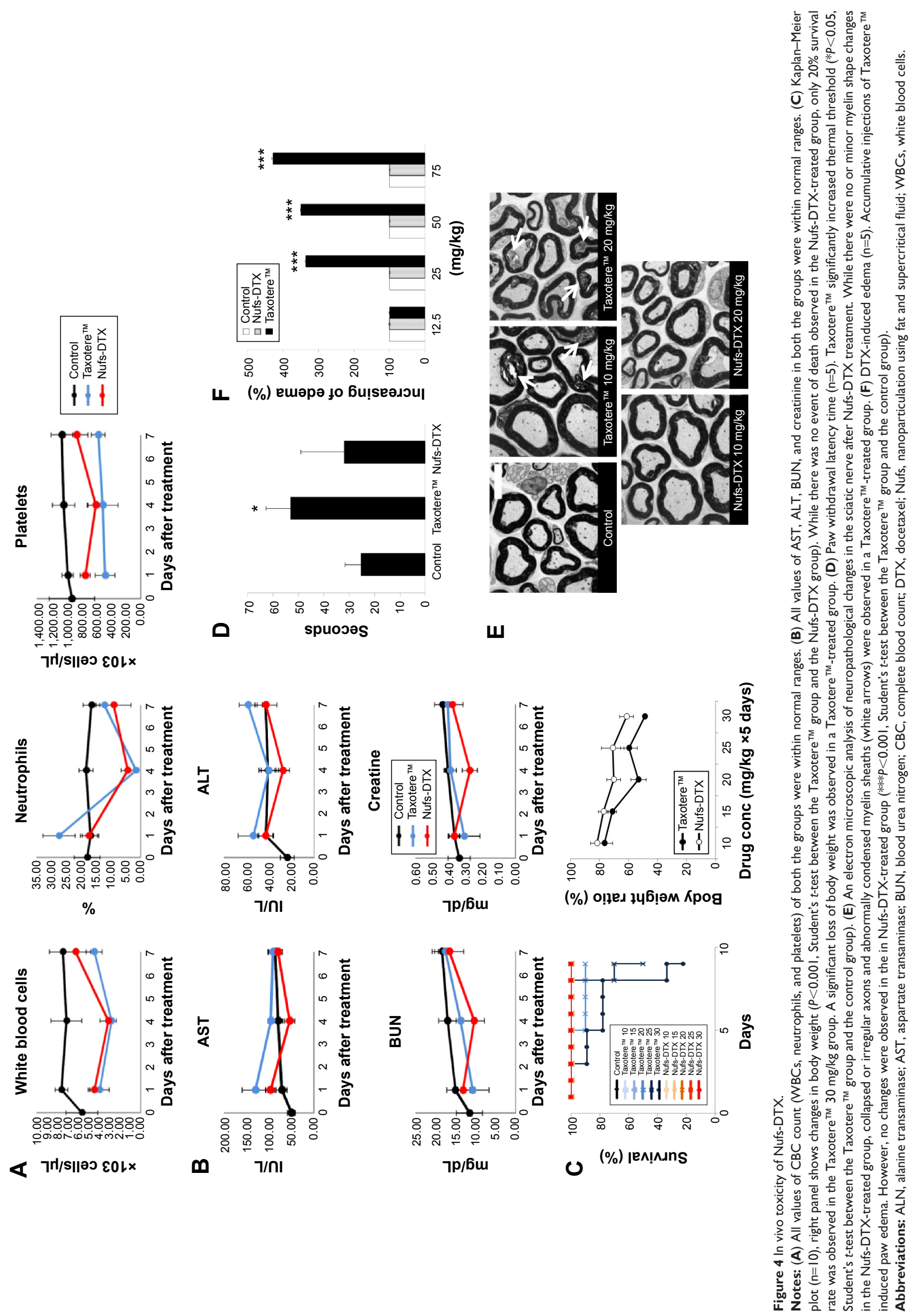

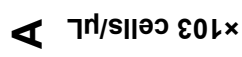

m

$7 / \mathrm{nI}$
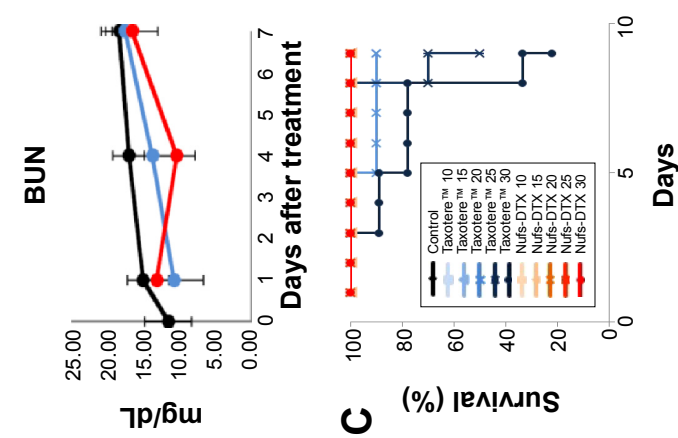

ง (\%) ןем!n.uns 
Table I Paralysis and survival rate after Nufs-DTX or Taxotere ${ }^{\mathrm{TM}}$ treatment

\begin{tabular}{llllll}
\hline $\begin{array}{lllll}\text { Dose } \\
\text { (mg/kg/day) }\end{array}$ & \multicolumn{2}{l}{ Paralysis (\%) } & & \multicolumn{2}{l}{ Survival (\%) } \\
\cline { 2 - 3 } \cline { 5 - 6 } \cline { 5 - 6 } & Taxotere & Nufs-DTX & & Taxotere & Nufs-DTX \\
\hline 0 & 0 & 0 & 100 & 100 \\
10 & 0 & 0 & 100 & 100 \\
15 & 0 & 0 & 100 & 100 \\
20 & 33.4 & 0 & 90 & 100 \\
25 & 60 & 0 & 50 & 100 \\
30 & 100 & 0 & 20 & 100
\end{tabular}

Abbreviations: DTX, docetaxel; Nufs, nanoparticulation using fat and supercritical fluid.

group (30 mg/kg/day) started to die from day 3, and the group's survival rate was $20 \%$ by day 10 (Table 1 and Figure 4C). On the other hand, no mice died in the Nufs-DTX-treated group as in the control group. The body weight was significantly decreased in the Taxotere ${ }^{\mathrm{TM}}$ group compared with the NufsDTX-treated group (Figure 4C). Moreover, motility was significantly reduced (data not shown) and waist-down paralysis was observed in the Taxotere ${ }^{\text {TM}}$-treated group $(20 \mathrm{mg} / \mathrm{kg} / \mathrm{day})$ (Table 1). Additionally, piloerection, hair loss, and reduction in food intake were observed in the Taxotere ${ }^{\mathrm{TM}}$-treated group (20 mg/kg/day). These toxicities were also observed in the Nufs-DTX-treated group (only $30 \mathrm{mg} / \mathrm{kg} /$ day) but to a lesser extent than that observed in the Taxotere ${ }^{\mathrm{TM}}$-treated group, indicating that Nufs-DTX had diminished DTX-induced toxicity (data not shown). In a thermal threshold test, we utilized a lower dose $(10 \mathrm{mg} / \mathrm{kg} /$ day $)$ to exclude general toxicity. Referring to a previous study, ${ }^{27}$ Nufs-DTX or Taxotere ${ }^{\mathrm{TM}}$ was used five times every day and then paw withdrawal latency time was measured at day 8 . Taxotere ${ }^{\mathrm{TM}}$ induced a significant increase (53.2 seconds) in thermal threshold compared with the control (25.5 seconds) (Figure 4D), while the increase in withdrawal latency in the Nufs-DTX-treated group was insignificant. This result suggests that Nufs-DTX reduces Taxotere ${ }^{\mathrm{TM}}$-induced hypoalgesia. To assess DTX-induced peripheral neuropathy, thermal threshold was examined among several experimental models. ${ }^{28}$ Taxotere ${ }^{\mathrm{TM}}$ showed abnormal algesia compared with the control from radiant heat (Figure 4D). We also performed an electron microscopic analysis for the observation of neuropathological changes in the sciatic nerve after iv injection of agents five times every day at a concentration of 10 or $20 \mathrm{mg} / \mathrm{kg}$. Normal myelinated fibers were observed in the control group (Figure 4E), while the sciatic nerve of mice treated with 10 or $20 \mathrm{mg} / \mathrm{kg}$ of Taxotere $^{\mathrm{TM}}$ revealed collapsed axons (white arrow, Figure 4E) surrounded by abnormal condensed multilamellar myelin sheaths and irregular shape of axons rather than the usual round shape found in control group (Figure 4E), as shown in a previous study. ${ }^{29}$ On the other hand, Nufs-DTX had attenuated DTX-induced neuropathy without any adjuvants (Figure 4E). These results indicate that Taxotere ${ }^{\mathrm{TM}}$ induced paralysis and hypoalgesia via axonopathy, but Nufs-DTX had reduced axonopathy-induced side effects.

Severe edema is one of the DTX-induced complications ${ }^{26}$ and affects the patients' quality of life. ${ }^{30}$ Clinical cases ${ }^{30}$ and indirect measurements ${ }^{31}$ were reported regarding DTX-induced edema. However, no animal models have been reported for the evaluation of edema. In this study, we established a DTX-induced edema model by repeatedly injecting agents at a high dose. This model could be a good evaluation tool as it uses the same administration routine as in clinic. As expected, edema was increased by $33.5 \%, 35.0 \%$, and $42.8 \%$ in the Taxotere ${ }^{\mathrm{TM}}$-treated groups $(25,50$, and $75 \mathrm{mg} / \mathrm{kg}$, respectively) compared with the control groups that were treated with the same volume of saline (Figure 4F). Surprisingly, no significant differences were observed in the Nufs-DTXtreated groups compared with the control groups.

Taken together, there were no observable toxicities in the blood samples of the Nufs-DTX-and Taxotere ${ }^{\text {TM}}$-treated groups at a given dose $(10 \mathrm{mg} / \mathrm{kg}$, single treatment in rats) (Figure $4 \mathrm{~A}$ and B). However, we were able to observe distinguishable toxicity responses such as reduced motility, waist-down paralysis, loss of body weight, abnormal algesia, degeneration of sciatic nerve, severe edema, and decreased survival rate after repeated treatments with high concentrations of drugs, whereas relatively low toxicity responses were observed in the Nufs-DTX-treated group (Figure 4C-F). Overall, these results indicate that nanoparticulated Nufs-DTX has significant therapeutic potential with no or less toxicity, relative to Taxotere ${ }^{\mathrm{TM}}$, with an efficient accumulation of drug in solid tumor tissues.

\section{Discussion}

It is known that solid nanosuspensions that are iv injected are mainly captured in the lungs, liver, and spleen via the reticuloendothelial system, followed by sustained release. From our biodistribution study results, we noticed that Nufs-DTX is mainly accumulated in the liver after injection and rapidly cleared within 30 minutes post-injection unlike Taxotere $^{\mathrm{TM}}$, which is mostly accumulated in the lungs and shows sustained release. Several studies suggested that the differences among main captured organs in biodistribution study might be dependent on the type of surface and size of nanosuspensions. ${ }^{32-34} \mathrm{We}$ are further investigating to solve the cause for differences of captured site using tracking and imaging system for Nufs-DTX. 
We also observed higher accumulation of DTX in the tumors of the Nufs-DTX-treated group compared with the Taxotere ${ }^{\mathrm{TM}}$-treated group (Figure $3 \mathrm{~A}$ and $\mathrm{B}$ ). However, the tumor growth inhibition efficiency did not meet our expectations. This might be caused by insufficient solubilization of Nufs-DTX in the tumors. Our next question is "How Nufs-DTX is solubilized in vivo and when Nufs-DTX solubilization occurs with the most effective efficacy"? We are currently focusing on solving these problematic issues.

In spite of several additional studies, Nufs-DTX produced by NUFS ${ }^{\mathrm{TM}}$ technology has remarkably improved aqueous dissolution rate, resulting in increased aqueous stability and bioavailability.

\section{Conclusion}

NUFS ${ }^{\mathrm{TM}}$ technology, which uses solid lipids as a solvent for active pharmaceutical ingredients and $\mathrm{CO}_{2}$ as a supercritical fluid for extracting lipids from solid mixtures, is an improved method that generates optimized delivery solution for poorly soluble drugs. In the perspective of biomedicine, this technology suggests not only a costeffective and simple but efficient and effective process in a manufacturer's perspective, but also a wide applicability and safety using environmentally friendly materials such as lipids and $\mathrm{CO}_{2}$. In this study, NUFS ${ }^{\mathrm{TM}}$ technology was successfully applied to generate homogenous nanoparticulated DTX with an average diameter of $170 \mathrm{~nm}$. We showed that homogenous Nufs-DTX in water possessed a number of significant advantages such as increased aqueous stability and bioavailability, increased tumor targeting and accumulation, and reduced toxicity with the preserved biochemical mechanism of Taxotere ${ }^{\mathrm{TM}}$. With extended screenings of other insoluble compounds, there is no doubt that NUFS ${ }^{\text {TM }}$ technology will provide lots of opportunities and challenges to the clinical and pharmaceutical industry.

\section{Acknowledgment}

This work was supported by a grant from the Korean Health Technology R\&D Project, Ministry for Health and Welfare, Republic of Korea (HI06C0868 and HI10C2014), and the National Research Foundation of Korea (NRF) grant funded by the Korean Government (MEST) (NRF2012R1A2A2A01014671 and NRF-2013R1A1A2011346).

\section{Disclosure}

The authors report no conflicts of interest in this work.

\section{References}

1. De Jong WH, Borm PJ. Drug delivery and nanoparticles: applications and hazards. Int J Nanomedicine. 2008;3(2):133-149.

2. Farokhzad OC, Langer R. Impact of nanotechnology on drug delivery. ACS Nano. 2009;3(1):16-20.

3. Kipp JE. The role of solid nanoparticle technology in the parenteral delivery of poorly water-soluble drugs. Int J Pharm. 2004;284(1-2): 109-122.

4. Hu J, Johnston KP, Williams RO 3rd. Nanoparticle engineering processes for enhancing the dissolution rates of poorly water soluble drugs. Drug Dev Ind Pharm. 2004;30(3):233-245.

5. Lee SJ, Kim YH, Lee SH, Hahn M. Characterization of nano oxaliplatin prepared by novel Fat Employing Supercritical Nano System, the FESNS(R). Pharm Dev Technol. 2012;17(2):212-218.

6. Zhao M, Su M, Lin X, et al. Evaluation of docetaxel-loaded intravenous lipid emulsion: pharmacokinetics, tissue distribution, antitumor activity, safety and toxicity. Pharm Res. 2010;27(8):1687-1702.

7. Fauzee NJ. Taxanes: promising anti-cancer drugs. Asian Pac J Cancer Prev. 2011;12(4):837-851.

8. Clarke SJ, Rivory LP. Clinical pharmacokinetics of docetaxel. Clin Pharmacokinet. 1999;36(2):99-114

9. Verweij J, Clavel M, Chevalier B. Paclitaxel (Taxol) and docetaxel (Taxotere): not simply two of a kind. Ann Oncol. 1994;5(6): 495-505.

10. Nel A, Xia T, Madler L, Li N. Toxic potential of materials at the nanolevel. Science. 2006;311(5761):622-627.

11. Fan AM, Alexeeff G. Nanotechnology and nanomaterials: toxicology, risk assessment, and regulations. J Nanosci Nanotechnol. 2010; 10(12):8646-8657.

12. Linkov I, Satterstrom FK, Corey LM. Nanotoxicology and nanomedicine: making hard decisions. Nanomedicine. 2008;4(2):167-171.

13. Wang Y, Chen L, Tan L, et al. PEG-PCL based micelle hydrogels as oral docetaxel delivery systems for breast cancer therapy. Biomaterials. 2014;35(25):6972-6985.

14. Wang Y, Wang C, Gong C, et al. Polysorbate 80 coated poly (varepsiloncaprolactone)-poly (ethylene glycol)-poly (varepsilon-caprolactone) micelles for paclitaxel delivery. Int J Pharm. 2012;434(1-2):1-8.

15. Jung J, Park SJ, Chung HK, et al. Polymeric nanoparticles containing taxanes enhance chemoradiotherapeutic efficacy in non-small cell lung cancer. Int J Radiat Oncol Biol Phys. 2012;84(1):e77-e83.

16. Choi J, Kim HY, Ju EJ, et al. Use of macrophages to deliver therapeutic and imaging contrast agents to tumors. Biomaterials. 2012;33(16): 4195-4203.

17. Choi J, Woo H-N, Ju EJ, et al. Immunocytes as a biocarrier to delivery therapeutic and imaging contrast agents to tumors. $J$ Nanomater. 2012;2012. Available from: http://dx.doi.org/10.1155/2012/863704. Accessed September 4, 2015.

18. Woolfe G, Macdonald AD. The evaluation of the analgesic action of pethidine hydrochloride (Demerol). J Pharmacol Exp Ther. 1944; 80(3):300-307.

19. Bianchi R, Buyukakilli B, Brines M, et al. Erythropoietin both protects from and reverses experimental diabetic neuropathy. Proc Natl Acad Sci U S A. 2004;101(3):823-828.

20. Wang YX, Bowersox SS, Pettus M, Gao D. Antinociceptive properties of fenfluramine, a serotonin reuptake inhibitor, in a rat model of neuropathy. J Pharmacol Exp Ther. 1999;291(3):1008-1016.

21. Patil CR, Gadekar AR, Patel PN, Rambhade A, Surana SJ, Gaushal MH Dual effect of Toxicodendron pubescens on carrageenan induced paw edema in rats. Homeopathy. 2009;98(2):88-91.

22. Taurin S, Nehoff H, Greish K. Anticancer nanomedicine and tumor vascular permeability; where is the missing link? J Control Release. 2012; 164(3):265-275.

23. Fang J, Nakamura $H$, Maeda $H$. The EPR effect: unique features of tumor blood vessels for drug delivery, factors involved, and limitations and augmentation of the effect. Adv Drug Deliv Rev. 2011;63(3):136-151.

24. Greish K. Enhanced permeability and retention of macromolecular drugs in solid tumors: a reoal gate targeted anticancer nanomedicines. J Drug Target. 2007;15(7-8):457-464. 
25. Kudlowitz D, Muggia F. Defining risks of taxane neuropathy: insights from randomized clinical trials. Clin Cancer Res. 2013;19(17):4570-4577.

26. Gelmon K. The taxoids: paclitaxel and docetaxel. Lancet. 1994; 344(8932):1267-1272.

27. Parvathy SS, Masocha W. Matrix metalloproteinase inhibitor COL-3 prevents the development of paclitaxel-induced hyperalgesia in mice. Med Princ Pract. 2013;22(1):35-41.

28. Roglio I, Bianchi R, Camozzi F, et al. Docetaxel-induced peripheral neuropathy: protective effects of dihydroprogesterone and progesterone in an experimental model. J Peripher Nerv Syst. 2009;14(1):36-44.

29. Park SB, Krishnan AV, Lin CS, Goldstein D, Friedlander M, Kiernan MC. Mechanisms underlying chemotherapy-induced neurotoxicity and the potential for neuroprotective strategies. Curr Med Chem. 2008; 15(29):3081-3094.

30. Ohsumi S, Shimozuma K, Ohashi Y, et al. Subjective and objective assessment of edema during adjuvant chemotherapy for breast cancer using taxane-containing regimens in a randomized controlled trial: the National Surgical Adjuvant Study of Breast Cancer 02. Oncology. 2012;82(3):131-138.
31. Bronstad A, Berg A, Reed RK. Effects of the taxanes paclitaxel and docetaxel on edema formation and interstitial fluid pressure. Am J Physiol Heart Circ Physiol. 2004;287(2):H963-H968.

32. De Jong WH, Hagens WI, Krystek P, Burger MC, Sips AJ, Geertsma RE. Particle size-dependent organ distribution of gold nanoparticles after intravenous administration. Biomaterials. 2008;29(12):1912-1919.

33. Alexis F, Pridgen E, Molnar LK, Farokhzad OC. Factors affecting the clearance and biodistribution of polymeric nanoparticles. Mol Pharm. 2008;5(4):505-515.

34. Kumar R, Roy I, Ohulchanskky TY, et al. In vivo biodistribution and clearance studies using multimodal organically modified silica nanoparticles. ACS Nano. 2010;4(2):699-708.

\section{Publish your work in this journal}

The International Journal of Nanomedicine is an international, peerreviewed journal focusing on the application of nanotechnology in diagnostics, therapeutics, and drug delivery systems throughout the biomedical field. This journal is indexed on PubMed Central, MedLine, CAS, SciSearch $®$, Current Contents ${ }^{\circledR} /$ Clinical Medicine,
Journal Citation Reports/Science Edition, EMBase, Scopus and the Elsevier Bibliographic databases. The manuscript management system is completely online and includes a very quick and fair peer-review system, which is all easy to use. Visit http://www.dovepress.com/ testimonials.php to read real quotes from published authors. 